\title{
EFEITO DE CICLOS DE UMIDADE RELATIVA E TEMPERATURA DO AR NA RESISTÊNCIA DE JUNTAS COLADAS COM LÂMINAS DE Eucalyptus grandis W. Hill ex Maiden, Eucalyptus saligna Smith E CHAPAS DE FIBRA DE DENSIDADE MÉDIA (MDF) ${ }^{1}$
}

\author{
Benedito Rocha Vital², Antônio da Silva Maciel² e Ricardo Marius Della Lucia²
}

\begin{abstract}
RESUMO - Este trabalho teve como objetivo avaliar o efeito de ciclos de umidade relativa e temperatura do ar na resistência da linha de cola, em juntas coladas entre lâminas de madeira de Eucalyptus grandis, Eucalyptus saligna e lâminas de chapa de fibras de média densidade (MDF) cujas densidades foram iguais a 0,60, 0,66, e $0,72 \mathrm{~g} / \mathrm{cm}^{3}$, respectivamente. Foram empregados adesivos de poliacetato de média viscosidade e uréia-formaldeído nas gramaturas de $150,0 \mathrm{~g} / \mathrm{m}^{2}$ e $180,0 \mathrm{~g} / \mathrm{m}^{2}$, respectivamente, em face simples. O teor médio de umidade no momento da colagem foi igual a $14 \%$. Foram observadas diferenças significativas tanto na resistência da linha de cola quanto na percentagem de falha na madeira provocada pela composição das amostras, etapa de equilíbrio e tipo de adesivo. Os valores mais elevados de resistência foram observados nas juntas coladas com madeira de Eucalyptus saligna após a primeira etapa de equilíbrio, enquanto os menores valores ocorreram nas juntas combinando lâminas de eucalipto e MDF. Considerando apenas as lâminas de madeira, os valores mais elevados de falha na madeira foram observados nas juntas coladas entre lâminas de Eucalyptus grandis. Nas amostras compostas de madeira de eucalipto e lâminas de MDF, a porcentagem de falha foi total e o rompimento ocorreu exclusivamente no interior da chapa de MDF. Observou-se interação significativa entre a composição das amostras lâminas e o tipo de adesivo, em que os valores mais altos de resistência ocorreram nas juntas coladas com madeira de Eucalyptus saligna e adesivo à base de uréia-formaldeído.
\end{abstract}

Palavra-chave: Eucalyptus grandis, Eucalyptus saligna, MDF, ciclos de umidade, adesivos e adesão.

\section{AIR RELATIVE HUMIDITY AND TEMPERATURE CYCLE EFFECTS ON THE RESISTANCE OF GLUED JOINTS BETWEEN BOARDS OF Eucalyptus grandis, Eucalyptus saligna AND MEDIUM DENSITY FIBERBOARD (MDF)}

\begin{abstract}
The objectives of this work was to evaluate the effect of air relative humidity and air temperature cycles on glue line resistance of glued veneer joints of Eucalyptus grandis, Eucalyptus saligna and medium density fiberboard (MDF). Densities of Eucalyptus grandis, Eucalyptus saligna wood and MDF were equal to $0,60,0.66, e 0.72 \mathrm{~g} / \mathrm{cm}^{3}$ respectively. Medium viscosity polyacetate and urea-formaldehyde adhesives were applied at rate $150 \mathrm{~g} / \mathrm{cm}^{2}$ and $180 \mathrm{~g} / \mathrm{cm}^{2}$, respectively, on one face. Sample mean moisture content was $14 \%$. Relative humidity and temperature cycles affected both resistance and percentage of wood failure. Higher values of resistance were observed on the glue line between Eucalyptus saligna wood after equilibrium on the first cycle. The lowest values were always observed on the glue line between wood veneer and MDF. Considering
\end{abstract}

\footnotetext{
${ }^{1}$ Recebido em 05.07.2004 e aceito para publicação em 10.08.2005.

${ }^{2}$ Departamento de Engenharia Florestal da UFV - 36570-000 Viçosa-MG. E-mail: <bvital@ufv.br>.
} 
only wood, the highest wood percentage failure was observed among Eucalyptus grandis veneer samples. On the glue line between eucalyptus veneer and MDF the failure always occurred inside the MDF. Considering the interaction between samples composition and adhesive type, it was observed that the highest resistance occurred with Eucalyptus saligna veneer using urea-formaldehyde adhesive.

Keywords: Eucalyptus grandis, Eucalyptus saligna, MDF, Moisture cycles, Adhesives and Adhesion.

\section{INTRODUÇÃO}

Nos últimos anos, as madeiras de reflorestamento, particularmente as do gênero Eucalyptus, têm despertado grande interesse pelo potencial que apresentam como fonte de matéria-prima para a fabricação de móveis e a construção civil. Isso tem motivado vários trabalhos de pesquisa visando a uma melhor caracterização de suas propriedades (OLIVEIRA, 1998; DELLA LUCIA e VITAL, 1980; NOGUEIRA e LAHR, 1992; SILVA e OLIVEIRA, 2003). Dentre as diversas propriedades que devem ser avaliadas, uma das mais importantes é a capacidade de adesão dessas madeiras, uma vez que mais de $70 \%$ de todos os produtos confeccionados a partir da madeira utilizam algum tipo de adesivo em sua fabricação (MARRA, 1980). Já se conhece o comportamento das juntas coladas de muitas espécies de madeira, incluindo algumas do gênero Eucalyptus (FAMER, 1972; DELLA LUCIA e VITAL, 1989; NASCIMENTO et al., 2001; SERPA, 2001; DELLALUCIA e VITAL, 1981). Algumas colam bem, outras produzem juntas consideradas insatisfatórias.

A adesão entre dois substratos é um fenômeno complexo e envolve processos físicos e moleculares. De maneira geral, os modelos mais usados para explicar o fenômeno da adesão podem ser divididos em adesão mecânica e adesão específica (FOURCHE,1995). Em ambos os modelos, para que ocorra uma boa adesão é necessário um grau adequado de umedecimento da superfície do substrato pelo adesivo e sua conseqüente penetração nos interstícios da superfície, solidificação e suficiente flexibilidade da massa polimerizada. Certa flexibilidade é necessária, a fim de reduzir os efeitos das tensões ocasionadas por esforços localizados. A adesão mecânica é o resultado do engachamento mecânico de polímeros em poros ou asperezas presentes nas superfícies dos substratos e se caracteriza pela penetração espontânea do adesivo, por capilaridade, nas porosidades interiores do substrato, tomando o seu contorno e dificultando sua saída após a solidificação do adesivo. A adesão específica é o resultado da ação

R. Árvore, Viçosa-MG, v.29, n.5, p.801-807, 2005 de forças intermoleculares de naturezas primária e secundária, cujo efeito se manifesta nas regiões interfaciais dos substratos (FOURCHE, 1995).

A madeira é um material poroso, anisotrópico e higroscópico. A sua capacidade de adsorção de água deve-se, sobretudo, às hemiceluloses, seguidas das celuloses. É na celulose e principalmente na camada S2 da parede celular, de forte caráter hidrofílico, que a ação da adsorção mais intensamente se manifesta. Sabe-se que na parede celular a água ocupa somente os espaços entre as cristalites, nas zonas amorfas das microfibrilas de celulose. Nessas zonas, as ligações entre as cadeias celulósicas são muito mais fracas do que as correspondentes existentes nas regiões cristalinas, sendo os sítios disponíveis para ligação mais abundantes. A água, ao penetrar nessas zonas amorfas, provoca uma desorganização em sua estrutura, que se manifesta por aumento da superfície interna da parede celular, pelo surgimento de microporos transitórios, responsáveis pelo inchamento e retração da madeira de acordo com a umidade do ambiente.

A madeira, por sua capacidade de adsorver água, sob diferentes condições de umidade relativa e temperatura, adquire diferentes teores de umidade, levando a mesma amostra de madeira a diferentes massas e volumes. Em condições absolutamente secas, a madeira apresenta um volume mínimo, que aumenta conforme o teor de umidade também aumenta, atingindo o volume máximo na umidade de saturação das fibras, cujo valor médio é cerca de $28 \%$. Acima desse valor, a água ocorre na forma livre, não contribuindo para a variação dimensional da madeira (VITAL, 1984), entre outros.

Certa estabilidade dimensional ocorre quando a madeira atinge a umidade de equilíbrio higroscópico. Contudo, variações na umidade relativa e temperatura do meio ambiente provocam alterações na umidade de equilíbrio e consequientes alterações nas dimensões. Além desses fatores, a umidade de equilíbrio é afetada 
pela história da exposição, espécie de madeira e presença de extrativos; varia entre alburno e cerne de uma mesma espécie e pela ação de tensões mecânicas (GALVÃO e JANKOWSKY, 1985).

Nas condições brasileiras, o teor médio de equilíbrio é de aproximadamente $15 \%$, com algumas variações, dependendo de cada região e época do ano.

As variações dimensionais da madeira ocorrem de forma diferenciada, segundo cada um dos eixos que compõem sua estrutura. Em geral, observa-se que a variação na direção tangencial é, aproximadamente, duas vezes maior que na direção radial, sendo menor na direção longitudinal (PANSHIN e De ZEEUW, 1980). Essas diferenças se devem, basicamente, à orientação das microfibrilas em relação ao eixo longitudinal das células, influência dos raios, efeito do maior número de pontuações nas paredes radiais, à água adsorvida ou dessorvida nas regiões amorfas da celulose e à alternância dos lenhos inicial e tardio (GALVÃO e JANKOWSKY, 1985). Segundo Tsoumis (1991), a magnitude da contração e da expansão da madeira varia entre espécies, sendo maior em espécies com maior densidade.

As variações dimensionais anisotrópicas são indesejáveis para determinados usos da madeira (DURLO e MARCHIORI, 1992). Na colagem de madeiras, por exemplo, tais variações podem provocar tensões nas juntas coladas, reduzindo sua resistência nas condições de trabalho.

O objetivo deste estudo foi avaliar o efeito de ciclos de umidade relativa e temperatura do ar na resistência ao cisalhamento de juntas coladas com madeira de Eucalyptus grandis, Eucalyptus saligna e chapas de fibras de média densidade (MDF).

\section{MATERIAL E MÉTODOS}

As madeiras utilizadas no ensaio experimental foram obtidas de árvores de Eucalyptus grandis e Eucalyptus saligna, com 50 e 40 anos idade, e densidades básica iguais a $0,60 \mathrm{e} 0,66 \mathrm{~g} / \mathrm{cm}^{3}$, respectivamente, provenientes de plantios existentes em áreas do Campus da Universidade Federal de Viçosa. As toras foram desdobradas em tábuas com espessuras médias de $30 \mathrm{~mm}$, secadas ao ar e armazenadas no Laboratório de Painéis e Energia da Madeira. Foram, também, empregadas lâminas de MDF com densidade igual a $0,72 \mathrm{~g} / \mathrm{cm}^{3}$.

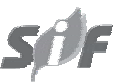

Foram utilizados pares de amostras na forma de lâminas de $600 \times 100 \times 9,5 \mathrm{~mm}$, tanto nas madeiras de eucalipto quanto no MDF. Os pares de lâminas de madeira sólida, bem como as amostras de MDF, foram levados à estufa, regulada a $35^{\circ} \mathrm{C}$, onde permaneceram até atingir umidade média de $14 \%$ para, posteriormente, serem coladas entre si. Pouco antes de receberem o adesivo, as superfícies das lâminas foram lixadas com lixa de grã 80 e limpadas com a finalidade de apresentar as faces ativadas. Alguns pares de lâminas foram separados, de forma a produzir amostras suficientes para compor os pares madeira/MDF, formando o arranjo apresentado no Quadro 1. Foram colados 72 pares, resultado de um arranjo fatorial completo do tipo 4 x 2 x $3 \times 3$, correspondente às combinações tipos de lâmina (espécie de madeira e MDF), tipos de adesivo, ciclos de umidade e repetições.

Os adesivos utilizados foram cedidos pela Alba Química, sendo aplicados segundo as recomendações especificadas nos respectivos Boletins Técnicos. Foram empregados adesivos em emulsão aquosa à base de poliacetato de vinila (PVAc) de média viscosidade (20004000 Cp) (CASCOREZ 2250) e resina sintética à base de uréia-formol, cura a frio (Cascamite PL-2030), apresentando um teor médio de sólidos igual a 50,0 e $65,0 \%$, respectivamente. Foram aplicados em face simples $150 \mathrm{~g} / \mathrm{m}^{2}$ do adesivo à base de poliacetato de vinila e $180 \mathrm{~g} / \mathrm{m}^{2}$ do adesivo à base de uréia-formaldeído. Os adesivos foram preparados poucos minutos antes de seu emprego, sendo espalhados sobre as superfícies com um pincel. O adesivo em emulsão foi homogeneizado e usado como adquirido. A formulação do adesivo à base de uréia-formaldeído foi preparada, misturando-se $100 \mathrm{~g}$ de resina, $20 \mathrm{~g}$ de farinha de trigo e $7 \mathrm{~g}$ de catalisador. Após a aplicação do adesivo, as juntas foram imediatamente fechadas e assim permaneceram por aproximadamente 15 minutos, sendo então levadas para a prensa. Aplicou-se uma pressão de $10 \mathrm{kgf} / \mathrm{cm}^{2}$ durante $8 \mathrm{~h}$, à temperatura de $23^{\circ} \mathrm{C}$.

Quadro 1 - Composição dos pares de lâminas na formação das juntas coladas

Table 1 - Veneer pair composition for glue lines

\begin{tabular}{lc}
\hline Eucalyptus grandis/Eucalyptus grandis & (Eg/Eg) \\
Eucalyptus saligna/Eucalyptus saligna & (Es/Es) \\
Eucalyptus grandis/MDF* & (Eg / MDF) \\
Eucalyptus saligna/MDF & (Es / MDF) \\
\hline
\end{tabular}

* MDF = painel de fibra de média densidade.

R. Árvore, Viçosa-MG, v.29, n.5, p.801-807, 2005 
Após a cura do adesivo, as juntas coladas foram deixadas em repouso, durante uma semana, em ambiente climatizado a $23 \pm 2{ }^{\circ} \mathrm{Ce} 75 \pm 3 \%$ de umidade, sendo depois desdobradas em corpos-de-prova nas dimensões especificadas na norma ASTM D 2339-94.

Os ciclos de umidade e temperatura foram fixados entre $50 \%$ e $25^{\circ} \mathrm{Ce} 80 \%$ e $30^{\circ} \mathrm{C}$, respectivamente. Assim, todos os corpos-de-prova foram colocados em uma câmara climática com umidade relativa igual a $50 \%$ e temperatura igual a $15{ }^{\circ} \mathrm{C}$, em que permaneceram até o equilíbrio. Foram, então, retiradas seis amostras de cada par de composição, totalizando 24 pares, que tiveram a sua resistência ao cisalhamento na linha de cola e a porcentagem de falhas na madeira determinadas. Nas demais amostras, a temperatura e umidade relativa foram elevadas para $30{ }^{\circ} \mathrm{C}$ e $80 \%$, respectivamente, em que permaneceram até o equilíbrio, observando-se valores médios iguais a 17,$20 ; 15,50 ; 14,70 ;$ e $12,80 \%$, nos pares de lâminas de Eucalyptus grandis, Eucalyptus saligna, Eucalyptus grandis/MDF e Eucalyptus saligna/MDF, respectivamente. Atingido o equilíbrio nessas condições, a câmara foi regulada, novamente, para $50 \%$ de umidade relativa e $15^{\circ} \mathrm{C}$ de temperatura, permanecendo nessas condições até os corpos-de-prova atingirem novamente o equilíbrio. Foram retiradas seis amostras de cada par de combinações, que teve suas propriedades determinadas. O restante das amostras foi submetido a novo ciclo de temperatura/umidade relativa, sendo as propriedades determinadas após o equilíbrio a $15^{\circ} \mathrm{C}$ e $50 \%$.

As resistências ao cisalhamento, em tração, foram determinadas segundo a norma ASTM D 2339-94. Na determinação de falhas na madeira, consideraram-se apenas as falhas profundas.

O resultado dos testes das variáveis em estudo foi submetido à análise de variância (ANOVA), sendo as médias comparadas pelo teste de Tukey a 5\% de probabilidade.

\section{RESULTADOS E DISCUSSÃO}

A avaliação do efeito dos tratamentos na umidade de equilíbrio, com o auxílio da análise de variância, indicou que houve diferença significativa oriunda da composição das amostras e da interação entre composição de amostras e etapas de equilíbrio. Os efeitos foram desdobrados, e os valores médios de umidade em cada composição das amostras em função da etapa de equilíbrio, com o respectivo teste de médias, são apresentados nos Quadro 2.

R. Árvore, Viçosa-MG, v.29, n.5, p.801-807, 2005
Quadro 2 - Valores médios de umidade após o equilíbrio na temperatura de $15^{\circ} \mathrm{C}$ e $50 \%$ de umidade relativa em função da etapa de equilíbrio

Table 2 -Moisture content mean values after equilibrium at $15^{\circ} \mathrm{C}$ and $50 \%$ relative humidity as a function of the equilibrium fase

\begin{tabular}{lccc}
\hline Composição da Amostra* & \multicolumn{2}{c}{ Etapa de Equilíbrio } \\
\cline { 2 - 4 } & 1 & 2 & 3 \\
\hline Es/Es & $11,91 \mathrm{Cb}^{* *}$ & $13,21 \mathrm{Bb}$ & $13,92 \mathrm{Ab}$ \\
$\mathrm{Eg} / \mathrm{Eg}$ & $12,06 \mathrm{Bb}$ & $15,00 \mathrm{Aa}$ & $15,03 \mathrm{Aa}$ \\
$\mathrm{Es} / \mathrm{MDF}$ & $11,76 \mathrm{Ab}$ & $11,56 \mathrm{Ad}$ & $11,76 \mathrm{Ad}$ \\
$\mathrm{Eg} / \mathrm{MDF}$ & $12,68 \mathrm{Aa}$ & $12,27 \mathrm{Ac}$ & $12,75 \mathrm{Ac}$ \\
$\mathrm{CV}(\%)$ & 6,12 & 11,45 & 10,34 \\
\hline * Em que: Es = Eucalyptus saligna, Eg = Eucalyptus grandis e & \\
MDF = chapa de fibra de densidade média. & \\
$* *$ Médias seguidas pela mesma letra maiúscula ao longo das linhas \\
ou pela mesma letra minúscula ao longo das colunas não diferem \\
entre si, pelo teste de Tukey no nível de 5\% de probabilidade.
\end{tabular}

Analisando o Quadro 2, observa-se que nas amostras constituídas por apenas madeira de Eucalyptus saligna a umidade de equilíbrio aumentou, de forma significativa, nas etapas sucessivas de equilíbrio. $\mathrm{Na}$ madeira de Eucalyptus grandis, a umidade de equilíbrio nas etapas 2 e 3 foram significativamente maiores daquela obtida na primeira etapa. Diferenças na umidade de equilíbrio, na desorção em relação à umidade de equilíbrio na adsorção, nas mesmas condições de temperatura e umidade, são comuns em madeiras, sendo esse fenômeno denominado histerese (SKAAR, 1972; 1988). As amostras compostas por chapas de MDF não tiveram o valor médio de equilíbrio higroscópico afetado pelas etapas de equilíbrio. Isso, provavelmente, é consequiência da elevada temperatura empregada em algumas etapas de fabricação das chapas de compósitos e que reduzem a sua higroscopicidade (MEYERS e McNATT, 1985).

Comparando as médias por composição de amostra dentro de cada etapa, observou-se que as amostras contendo madeira de Eucalyptus grandis, de modo geral, entraram em equilíbrio com teores de umidade mais elevados.

Analisando a média geral no Quadro 3, observase que as juntas coladas com madeira de Eucalyptus saligna foram as que proporcionaram os valores mais elevados de resistência ao cisalhamento, diferindo estatisticamente dos valores observados nas lâminas coladas com madeira de Eucalyptus grandis. Os resultados apresentados revelam ainda que, independentemente da espécie de eucalipto utilizada para formar as juntas com lâminas de MDF, os menores 
valores de resistência foram determinados nessas combinações. Tal fato já era esperado, pois se sabe que a madeira reconstituída, por suas características intrínsecas, apresenta resistência inferior aos esforços cisalhantes, em comparação com os valores determinados na madeira sólida.

A análise de variância das médias de resistência ao cisalhamento indicou diferenças significativas entre composição das amostras, tipo de adesivo e etapa de equilíbrio. A interação entre composição das amostras e tipo de adesivo foi significativa. A porcentagem de falhas na madeira não foi afetada pela etapa de equilíbrio, mas sim pela composição das amostras e pelo tipo de adesivo. A interação entre esses dois tratamentos foi significativa.

A menor porcentagem de falha na madeira foi observada nas juntas coladas com madeira de Eucalyptus saligna. A resistência ao cisalhamento dessa madeira é mais elevada do que a da madeira de Eucalyptus grandis (SERPA, 2001) e, provavelmente, próxima da resistência oferecida pelos adesivos, uma vez que houve redução significativa da porcentagem de falha na madeira e, conseqüentemente, aumento na porcentagem de falha ocorrida na linha de cola.

Nas juntas coladas com a combinação de lâminas de madeira e de MDF não se observou diferença significativa entre os valores médios determinados, independentemente da espécie de eucalipto utilizada. Observou-se, contudo, que a falha sempre ocorreu no MDF. Assim, a redução significativa na tensão de

Quadro 3 - Valores médios da resistência ao cisalhamento da linha de cola $\left(\mathrm{kgf} / \mathrm{cm}^{2}\right)$ e porcentagem de falha (\%), segundo a combinação de lâminas

Table 3 - Glue line shear resistance $\left(\mathrm{kgf} / \mathrm{cm}^{2}\right)$ mean values and percentage of wood failure according to sample composition

\begin{tabular}{|c|c|c|}
\hline $\begin{array}{l}\text { Composição } \\
\text { das Amostras* }\end{array}$ & $\begin{array}{c}\text { Tensão de } \\
* \text { Ruptura }\left(\mathrm{kgf} / \mathrm{cm}^{2}\right)\end{array}$ & $\begin{array}{c}\text { Falha na } \\
\text { Madeira (\%) }\end{array}$ \\
\hline Es/Es & $97,89 \mathrm{~A} * *(7,09) * * *$ & $39,61 \mathrm{C}(38,45)$ \\
\hline $\mathrm{Eg} / \mathrm{Eg}$ & 86,59 В $(5,90)$ & 63,55 В $(13,09)$ \\
\hline Es/MDF & $30,61 \mathrm{C}(7,19)$ & $100,00 \mathrm{~A}(0,00)$ \\
\hline $\mathrm{Eg} / \mathrm{MDF}$ & $29,45, \mathrm{C}(5,33)$ & 100,00 A $(0,00)$ \\
\hline
\end{tabular}

* Em que: Es = Eucalyptus saligna, Eg = Eucalyptus grandis $\mathrm{e}$ MDF = chapa de fibra de média densidade.

** Em cada coluna, médias seguidas pela mesma letra não diferem estatisticamente, pelo teste de Tukey a $5 \%$ de probabilidade.

*** Coeficiente de variação. ruptura e $100 \%$ de falha sem diferença significativa entre amostras compostas de eucalipto e MDF indicam que houve adesão adequada e com resistência superior à resistência intrínseca do MDF, boa adesão entre a madeira e o MDF.

No Quadro 4, mostra-se a influência das etapas de equilíbrio na resistência das juntas coladas. A porcentagem de falha na madeira não foi afetada pelas etapas de equilíbrio. Contudo, observa-se, nesse quadro, que a resistência ao cisalhamento diminuiu significativamente entre a primeira, segunda e terceira etapas de equilíbrio. A redução na resistência da linha de cola pode ser decorrente da maior umidade de equilíbrio higroscópico e pode, ainda, ser um indício de que as variações dimensionais provocadas pelas alterações na umidade de equilíbrio geraram tensões que foram suficientes para produzir essa redução.

No Quadro 5 são apresentadas as médias de resistência ao cisalhamento e porcentagem de falha na madeira, resultantes do efeito da interação entre os fatores composição das juntas coladas e tipos de adesivos aplicados.

Observou-se que a resistência ao cisalhamento e a porcentagem de falha na madeira foram influenciados pela composição das juntas e pelo tipo de adesivo. Em cada tipo de adesivo, as lâminas coladas com madeira de Eucalyptus saligna apresentaram valores médios de resistência superiores aos encontrados nas lâminas de Eucalyptus grandis. Esses resultados são coerentes com os valores encontrados na literatura por Della Lucia e Vital (1981).

Quadro 4 - Influência das etapas de equilíbrio na resistência da linha de cola $\left(\mathrm{kgf} / \mathrm{cm}^{2}\right)$ e porcentagem de falha na madeira $(\%)$

Table 4-Effect of the equilibrium fase on the glue line resistance $\left(\mathrm{kgf} / \mathrm{cm}^{2}\right)$ and percentage of wood failure (\%)

\begin{tabular}{ccc}
\hline $\begin{array}{c}\text { Etapa de } \\
\text { equilíbrio }\end{array}$ & $\begin{array}{c}\text { Tensão de } \\
\text { Ruptura }\left(\mathrm{kgf} / \mathrm{cm}^{2}\right)\end{array}$ & $\begin{array}{c}\text { Falha } \\
\text { na madeira }\end{array}$ \\
\hline 1 & $63,63 \mathrm{~A}^{*}(51,50)^{* *}$ & $76,50(34,35)$ \\
2 & $59,97 \mathrm{~B}(53,76)$ & $74,31(38,95)$ \\
3 & $59,80 \mathrm{~B}(52,98)$ & $76,56(36,18)$ \\
\hline
\end{tabular}

*Em cada coluna, médias seguidas pela mesma letra não diferem estatisticamente, pelo teste de Tukey no nível de $5 \%$ de probabilidade. ** Coeficiente de variação.

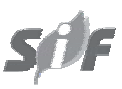

R. Árvore, Viçosa-MG, v.29, n.5, p.801-807, 2005 
Quadro 5 - Valores médios da resistência da linha de cola $\left(\mathrm{kgf} / \mathrm{cm}^{2}\right)$ e porcentagem de falha na madeira $(\%)$, resultantes da interação entre a composição das amostras e o tipo de adesivo

Table 5 -Mean values for glue line resistance and percentage of wood failure when the interaction between sample composition and adhesives type is considered

\begin{tabular}{|c|c|c|c|c|}
\hline \multirow[t]{2}{*}{ Composição das Amostras* } & \multicolumn{2}{|c|}{ Tensão de Ruptura $\left(\mathrm{kgf} / \mathrm{cm}^{2}\right)$} & \multicolumn{2}{|c|}{ Falha na Madeira (\%) } \\
\hline & PVAc & Uréia-Formaldeído & PVAc & Uréia-Formaldeído \\
\hline Es / Es & $95,07 \mathrm{~A} \mathrm{~b} * *(7,02) * * *$ & 100,72 Аа $(6,26)$ & $30,33 \mathrm{Cb}(22,58)$ & $48,89 \mathrm{Ca}(32,48)$ \\
\hline $\mathrm{Eg} / \mathrm{Eg}$ & $83,04 \mathrm{Bb}(4,52)$ & $90,14 \quad \mathrm{Ba}(4,00)$ & $61,06 \mathrm{Ba}(14,95)$ & $66,06 \mathrm{Ba}(10,67)$ \\
\hline Es / MDF & $30,89 \mathrm{Ca}(6,96)$ & $\mathrm{Ca}(7,72)$ & 100,00 Аа $(0,00)$ & 100,00 Аа $(0,00)$ \\
\hline $\mathrm{Eg} / \mathrm{MDF}$ & $29,56 \mathrm{Ca}(4,50)$ & $\mathrm{Ca}(6,33)$ & 100,00 Аа $(0,00)$ & $100,00 \mathrm{Aa}$ \\
\hline
\end{tabular}

* Em que: Es = Eucalyptus saligna $; \mathrm{Eg}=$ Eucalyptus grandis $; \mathrm{MDF}=\mathrm{Chapa}$ de fibra de densidade média

** Médias seguidas pela mesma letra maiúscula ao longo das colunas ou pela mesma letra minúscula ao longo das linhas não diferem entre si, pelo teste de Tukey no nível de $5 \%$ de probabilidade.

*** Coeficiente de variação.

Nas juntas coladas com madeiras de eucalipto e MDF não houve efeito significativo da espécie de madeira na resistência das juntas, independentemente do tipo de adesivo utilizado. Os valores médios de resistência nessas combinações foram inferiores àqueles determinados em corpos-de-prova oriundos de lâminas de madeira de eucalipto, e isso se deve às características de menor resistência ao esforço cisalhante apresentado pelas lâminas de madeira reconstituída, em comparação com a da madeira sólida.

A análise estatística da porcentagem de falha na madeira indicou que os menores valores foram obtidos nas juntas coladas de madeira de Eucalyptus saligna, independentemente do tipo de adesivo empregado, embora numericamente se tenham observado valores mais elevados de falha na madeira quando se utilizou o adesivo de uréia-formaldeído. Nas combinações de madeira com MDF, não houve diferença significativa nesse parâmetro, ressaltando-se que todos os corposde-prova se romperam, exclusivamente, na lâmina de fibras.

Apesar das controvérsias, o porcentual de falha na madeira é um importante parâmetro nos testes de adesão. Elevados valores para o porcentual de falha na madeira são indicativos de boa qualidade na adesão, pois indicam que a coesão do adesivo e a resistência da interface adesivo/madeira são iguais ou mais elevadas que a resistência da própria madeira.

No presente estudo, nas juntas coladas com lâminas de madeira os valores determinados nessa propriedade foram, de modo geral, inferiores aos encontrados na literatura. Contudo, deve-se ressaltar que foram quantificadas, exclusivamente, as falhas profundas na madeira ou no MDF.

\section{CONCLUSÕES}

A exposição a sucessivos ciclos de umidade ocasionou aumento na umidade de equilíbrio higroscópico na dessorção das amostras constituídas apenas de madeira sólida. Amostras contendo MDF não tiveram o seu teor de equilíbrio afetado.

A resistência ao cisalhamento, bem como a porcentagem de falha na madeira, foi afetada pela composição das amostras. As amostras mais resistentes foram aquelas formadas com madeira de Eucalyptus saligna, porém com menor porcentagem de falha profunda na madeira.

A exposição a sucessivos ciclos de umidade provocou redução na resistência da linha de cola, porém não afetou a porcentagem de falha na madeira.

A tensão de ruptura na linha de cola, bem como a percentagem de falha na madeira das amostras contendo apenas madeira sólida, foi maior quando se empregou o adesivo à base de uréia-formaldeído. Nas amostras contendo MDF, o tipo de adesivo não afetou essas características.

\section{REFERÊNCIAS BIBLIOGRÁFICAS}

AMERICAN SOCIETY FOR TESTING AND

MATERIALS - ASTM. Annual book of

ASTM standards. Philadelphia: 1994. v. 15.

$478 \mathrm{p}$. 
DELLA LUCIA, R. M.; VITAL, B. R.

Características físicas e mecânicas da madeira de Eucalyptys grandis W. Hill ex Maiden. Revista Árvore, v. 4, n. 1, p. 70-74, 1980.

DELLA LUCIA, R. M.; VITAL, B. R. Avaliação da qualidade de juntas coladas de Madeira de 3 espécies de Eucalyptus. Revista Árvore, v. 5, n. 2, p. 172-180, 1981.

DELLA LUCIA, R. M.; VITAL, B. R. Ensaio de adesão das madeiras de Eucalyptus gummifera, E. paniculata e E. resinifera. Revista Árvore, v.13, n. 1, p. 98-106, 1989.

DURLO, M. A.; MARCHIORI, J. N. C. Tecnología da madeira: retratibilidade Santa Maria, CEPEF/ FATEC, 1992. 33 p. (Série Técnica, 10)

FAMER, R. M. Handbook of hardwoods. London, Her Majesty's Stationery Office, 1972. 244p.

FOURCHE, G. An overview of the basic aspects of polymer adhesion.Part1. Fundamentals. Polymer Engineering and Science, v. 35, n. 12, p.957. 1995.

GALVÃO, A. P. M.; JANKOWSKY, I. P.

Secagem racional da madeira. São Paulo: Nobel, 1985. 111 p.

MARRA,G. G. The role of adhesion and adhesives in products industry. In: Wood adhesives research, aplication and needs, 1980, Washington. Proceedings... Washington: 1980. p. $1-8$.

MEYERS, G.E.; McNATT, J.D. Fiberboard and hardboard research at Forest Products Laboratory - A 50-year summary. Madison: 1985. 39 p. (Gen. Tec. Report FPL 47. Forest Products Laboratory).

NASCIMENTO, A. M.; DELLA LUCCIA, R. M.; VITAL, B. R. Colagem de emendas biseladas em madeiras de Pinus spp. e Eucalyptus

citriodora. Revista Floresta e Ambiente, v. 8, p. 44-51, 2001.
NOGUEIRA, M. C. J. A .; LAHR, F. A. R. Indicações para o emprego de dezesseis espécies de Eucalyptus na construção civil. In: ENCONTRO BRASILEIRO EM MADEIRAS E EM ESTRUTURAS DE MADEIRA, 4., 1992. São Carlos. Anais... São Carlos: LaMEM-EESC-USP, 1992. v.I, p. 37-48.

OLIVEIRA, J. T. S. Caracterização da madeira de eucalipto para a construção civil. 1998. 429 f. Tese (Doutorado em Engenharia Civil) -Universidade de São Carlos, São Carlos, 1998.

PANSHIN, A. J.; DE ZEEUW, C. Textbook of wood technology. 4.ed. New York:Mc-GrawHill, 1980. 772 p.

SERPA, P.N. Avaliação da madeira de Eucalyptus grandis W. Hill ex Maiden, Eucalyptus saligna Smith e Pinus elliottii Engelm para a produção de móveis. 2001. 82 f. Dissertação (Mestrado em Ciência Florestal) - Universidade Federal de Viçosa, Viçosa, 2001.

SILVA, J. C.; OLIVEIRA, J. T. S. Avaliação das propriedades higroscópicas da madeira de Eucalyptus saligna $\mathrm{Sm}$., em diferentes condições de umidade relativa do ar. Revista Árvore, v. 27, n. 2, p. 233-239, 2003.

SKAAR, C. Wood-water relations. Berlin: Springer-Verlag, 1988. 283 p.

SKAAR, C. Water in wood. Syracuse: Syracuse University Press, 1972. 218 p.

TSOUMIS, G. Science and Technology of wood: structure, properties, utilization. New York: Van Nostrand Reinhold, 1991. 494 p.

VITAL, B. R. Métodos de determinação da densidade da Madeira. Viçosa:

Sociedade de Investigações Florestais, 1984. 21 p. (Boletim Técnico, 1). 